\title{
Rib 6
}

National Cancer Institute

\section{Source}

National Cancer Institute. Rib 6. NCI Thesaurus. Code C52762.

The sixth rib counting from the top of the rib cage down. 